Теорія Ймовір. та Матем. Статист. Вип. 74, 2006
Theor. Probability and Math. Statist.

No. 74, 2007, Pages 1-10

S 0094-9000(07)00692-8

Article electronically published on June 25, 2007

\title{
ASYMPTOTIC ANALYSIS OF A MEASURE OF VARIATION
}

\author{
UDC 519.21
}

\author{
H. ALBRECHER AND J. L. TEUGELS
}

\begin{abstract}
Let $X_{i}, i=1, \ldots, n$, be a sequence of positive independent identically distributed random variables and define

$$
T_{n}:=\frac{X_{1}^{2}+X_{2}^{2}+\cdots+X_{n}^{2}}{\left(X_{1}+X_{2}+\cdots+X_{n}\right)^{2}} .
$$

Utilizing Karamata's theory of functions of regular variation, we determine the asymptotic behaviour of arbitrary moments $\mathrm{E}\left(T_{n}^{k}\right), k \in \mathbb{N}$, for large $n$, given that $X_{1}$ satisfies a tail condition, akin to the domain of attraction condition from extreme value theory. As a by-product, the paper offers a new method for estimating the extreme value index of Pareto-type tails.
\end{abstract}

\section{INTRODUCTION}

Let $X_{i}, i=1, \ldots, n$, be a sequence of positive independent identically distributed (i.i.d.) random variables with distribution function $F$ and define

$$
T_{n}:=\frac{X_{1}^{2}+X_{2}^{2}+\cdots+X_{n}^{2}}{\left(X_{1}+X_{2}+\cdots+X_{n}\right)^{2}} .
$$

The asymptotic behaviour of $\mathrm{E}\left(T_{n}\right)$ was investigated in [5], simplifying and generalizing earlier results in [4] and 6].

In this paper we extend several results of [5] and derive the limiting behaviour of arbitrary moments

$$
\mathrm{E}\left(T_{n}^{k}\right), \quad k \in \mathbb{N} .
$$

This is achieved by using an integral representation of $\mathrm{E}\left(T_{n}^{k}\right)$ in terms of the Laplace transform of $X_{1}$, which is derived in Section 2.

Most of our results will be derived under the condition that $X_{1}$ satisfies

$$
1-F(x) \sim x^{-\alpha} \ell(x), \quad x \uparrow \infty,
$$

where $\alpha>0$ and $\ell(x)$ is slowly varying, i.e.

$$
\lim _{x \rightarrow \infty} \ell(t x) / \ell(x)=1
$$

for all $t>0$; see e.g. 3]. It is well known that condition (2) appears as the essential condition in the domain of attraction problem of extreme value theory. For a recent treatment, see [2]. A distribution satisfying (2) is called of Pareto-type with index $\alpha$.

2000 Mathematics Subject Classification. Primary 62G20; Secondary 62G32.

Key words and phrases. Functions of regular variation, domain of attraction of a stable law, extreme value theory.

Supported by Fellowship F/04/009 of the Katholieke Universiteit Leuven and the Austrian Science Foundation Project S-8308-MAT. 
When $\alpha<2$, then the condition coincides with the domain of attraction condition for weak convergence to a nonnormal stable law. It is then obvious that for $\beta>0$,

$$
\mathrm{E}\left(X_{1}^{\beta}\right):=\mu_{\beta}=\beta \int_{0}^{\infty} x^{\beta-1}(1-F(x)) d x \leq \infty
$$

will be finite if $\beta<\alpha$ but infinite whenever $\beta>\alpha$. For convenience, we define $\mu_{0}:=1$ and $\mu:=\mu_{1}$.

The results of this paper are based on the theory of functions of regular variation (see e.g. 3]). Clearly, if $\mathrm{E}\left(X_{1}\right)=\infty$, both the numerator and the denominator in (11) will exhibit an erratic behaviour, whereas for $\mathrm{E}\left(X_{1}\right)<\infty$ and $\mathrm{E}\left(X_{1}^{2}\right)=\infty$, this is the case only for the numerator. The results in Section 3 quantify this effect.

As a by-product, the results of this paper suggest a new method for estimating the extreme value index of Pareto-type distributions from a data set of observations, which is discussed in Section 4 .

The quantity $T_{n}$ is a basic ingredient in the study of the sample coefficient of variation of a given set of independent observations $X_{1}, \ldots, X_{n}$ from a random variable $X$, which is a frequently used risk measure in practical applications. In 11, this connection will be used to derive asymptotic properties of the sample coefficient of variation, including a distributional approach.

\section{Preliminaries}

Let $\varphi(s):=\mathrm{E}\left(e^{-s X_{1}}\right)=\int_{0}^{\infty} e^{-s x} d F(x), s \geq 0$, denote the Laplace transform of $X_{1}$. Then, following an idea of [5], one can use the identity

$$
\frac{1}{x^{\beta}}=\frac{1}{\Gamma(\beta)} \int_{0}^{\infty} e^{-s x} s^{\beta-1} d s, \quad \beta>0,
$$

and Fubini's theorem to deduce that

$$
\mathrm{E} \frac{1}{X_{1}^{\beta}}=\frac{1}{\Gamma(\beta)} \int_{0}^{\infty} s^{\beta-1} \varphi(s) d s .
$$

More generally, for i.i.d. random variables $X_{1}, \ldots, X_{n}$, one obtains the representation formula

$$
\mathrm{E} \frac{\prod_{i=1}^{n} X_{i}^{k_{i}}}{\left(X_{1}+X_{2}+\cdots+X_{n}\right)^{\beta}}=\frac{(-1)^{k_{1}+\cdots+k_{n}}}{\Gamma(\beta)} \int_{0}^{\infty} s^{\beta-1} \prod_{i=1}^{n} \frac{\partial^{k_{i}} \varphi(s)}{\partial s^{k_{i}}} d s,
$$

for nonnegative integers $k_{i}, i=1, \ldots, n$.

In particular, by symmetry,

$$
\mathrm{E}\left(T_{n}\right)=\mathrm{E} \frac{X_{1}^{2}+X_{2}^{2}+\cdots+X_{n}^{2}}{\left(X_{1}+X_{2}+\cdots+X_{n}\right)^{2}}=n \int_{0}^{\infty} s \varphi^{\prime \prime}(s) \varphi^{n-1}(s) d s,
$$

which formed the basis for the analysis in [5]. The representation (5) can be generalized in the following way:

Lemma 2.1. For an arbitrary positive integer $k$,

$$
\mathrm{E}\left(T_{n}^{k}\right)=\sum_{r=1}^{k} \sum_{\substack{k_{1}, \ldots, k_{r} \geq 1 \\ k_{1}+\cdots+k_{r}=k}} \frac{k !}{k_{1} ! \cdots k_{r} !} B\left(n, k_{1}, \ldots, k_{r}\right)
$$

with

$$
B\left(n, k_{1}, \ldots, k_{r}\right)=\frac{\left(\begin{array}{l}
n \\
r
\end{array}\right)}{\Gamma(2 k)} \int_{0}^{\infty} s^{2 k-1} \varphi^{\left(2 k_{1}\right)}(s) \cdots \varphi^{\left(2 k_{r}\right)}(s) \varphi^{n-r}(s) d s
$$


Proof. For an arbitrary positive integer $k$ we have

$$
\mathrm{E}\left(T_{n}^{k}\right)=\mathrm{E} \frac{\left(X_{1}^{2}+X_{2}^{2}+\cdots+X_{n}^{2}\right)^{k}}{\left(X_{1}+X_{2}+\cdots+X_{n}\right)^{2 k}}=\sum_{\substack{k_{1}, \ldots, k_{n} \geq 0 \\ k_{1}+\cdots+k_{n}=k}} \frac{k !}{k_{1} ! \cdots k_{n} !} \mathrm{E} \frac{X_{1}^{2 k_{1}} X_{2}^{2 k_{2}} \cdots X_{n}^{2 k_{n}}}{\left(X_{1}+X_{2}+\cdots+X_{n}\right)^{2 k}},
$$

where $k_{i} \leq k$ are nonnegative integers. Choose an $n$-tuple $\left(k_{1}, \ldots, k_{n}\right)$ in the above sum and let $r$ denote the number of its nonzero elements $\left(k_{i_{1}}, \ldots, k_{i_{r}}\right)$ (clearly $1 \leq r \leq k$ ). There are exactly $\left(\begin{array}{l}n \\ r\end{array}\right)$ possibilities of extending $\left(k_{i_{1}}, \ldots, k_{i_{r}}\right)$ to an $n$-tuple by filling in $n-r$ zeroes; each of the resulting $n$-tuples leads to the same summand in (66). Thus we can write

$$
\mathrm{E}\left(T_{n}^{k}\right)=\sum_{r=1}^{k} \sum_{\substack{k_{1}, \ldots, k_{r} \geq 1 \\
k_{1}+\cdots+k_{r}=k}} \frac{k !}{k_{1} ! \cdots k_{r} !} \underbrace{\left(\begin{array}{c}
n \\
r
\end{array}\right) \mathrm{E} \frac{X_{1}^{2 k_{1}} X_{2}^{2 k_{2}} \cdots X_{n}^{2 k_{r}}}{\left(X_{1}+X_{2}+\cdots+X_{n}\right)^{2 k}},}_{:=B\left(n, k_{1}, \ldots, k_{r}\right)}
$$

so that (6) holds in view of (44).

\section{MAin RESUlts}

As promised, we will assume in the sequel that $X_{1}$ satisfies condition (2). Recall that when $\alpha>1$, then $\mu<\infty$ while $\mu_{2}<\infty$ as soon as $\alpha>2$. The finiteness of $\mu$ and/or $\mu_{2}$ has its influence on the asymptotic behaviour of the summands that make up the statistic $T_{n}$. It is therefore not surprising that our results will be heavily dependent on the range of $\alpha$. We state a first and general result.

Lemma 3.1. If $X_{1}$ has a regularly varying tail with index $\alpha>0$, i.e.

$$
1-F(x) \sim x^{-\alpha} \ell(x),
$$

then the asymptotic behaviour of the $m$-th derivative of the Laplace transform $\varphi(s)$ as $s \downarrow 0$ is given by

$$
\varphi^{(m)}(s) \sim(-1)^{m} \alpha \Gamma(m-\alpha) s^{\alpha-m} \ell(1 / s), \quad m>\alpha .
$$

Proof. Let $\chi(s):=\int_{0}^{\infty} e^{-s x}(1-F(x)) d x$. Since $1-F(x) \sim x^{-\alpha} \ell(x)$, it follows that for $k>\alpha-1$,

$$
(-1)^{k} \chi^{(k)}(s)=\int_{0}^{\infty} x^{k} e^{-s x}(1-F(x)) d x \sim \Gamma(k+1-\alpha) s^{-k-1}\left(1-F\left(\frac{1}{s}\right)\right) \quad \text { as } s \rightarrow 0 .
$$

Since $\varphi(s)=1-s \chi(s)$, we have for $m \geq 1$,

$$
\varphi^{(m)}(s)=-m \chi^{(m-1)}(s)-s \chi^{(m)}(s),
$$

so that for $m>\alpha$,

$$
\begin{aligned}
\frac{s^{m} \varphi^{(m)}(s)}{1-F(1 / s)} & =-m \frac{s^{m} \chi^{(m-1)}(s)}{1-F(1 / s)}-\frac{s^{m+1} \chi^{(m)}(s)}{1-F(1 / s)} \\
& \sim(-1)^{m}(m \Gamma(m-\alpha)-\Gamma(m+1-\alpha))=(-1)^{m} \alpha \Gamma(m-\alpha),
\end{aligned}
$$

from which the assertion follows.

Theorem 3.1. If $X_{1}$ belongs to the domain of attraction of a stable law with index $\alpha$, $0<\alpha<1$, then for all $k \geq 1$,

$$
\lim _{n \rightarrow \infty} \mathrm{E}\left(T_{n}^{k}\right)=\frac{k !}{\Gamma(2 k)} \sum_{r=1}^{k} \frac{\alpha^{r-1}}{r \Gamma(1-\alpha)^{r}} G(r, k),
$$


where $G(r, k)$ is the coefficient of $x^{k}$ in the polynomial

$$
\left(\sum_{j=1}^{k-r+1} \frac{\Gamma(2 j-\alpha)}{j !} x^{j}\right)^{r}
$$

Proof. From $1-F(x) \sim x^{-\alpha} \ell(x)$ it follows that $1-\varphi(s) \sim \Gamma(1-\alpha) s^{\alpha} \ell(1 / s)$ (see e.g. Corollary 8.1.7 in [3]). Moreover, for any sequence $\left(a_{n}\right)_{n \geq 1}$ with $a_{n} \rightarrow \infty$ we have

$$
\begin{aligned}
\varphi^{n}\left(\frac{s}{a_{n}}\right) & =\exp \left\{n \log \varphi\left(s / a_{n}\right)\right\} \sim \exp \left\{-n\left(1-\varphi\left(s / a_{n}\right)\right)\right\} \\
& \sim \exp \left\{-n\left(\frac{s}{a_{n}}\right)^{\alpha} \ell\left(\frac{a_{n}}{s}\right) \Gamma(1-\alpha)\right\} .
\end{aligned}
$$

Choose $\left(a_{n}\right)_{n \geq 1}$ such that

$$
n a_{n}^{-\alpha} \ell\left(a_{n}\right) \Gamma(1-\alpha) \rightarrow 1 \quad \text { as } n \rightarrow \infty .
$$

Then for all $s \geq 0$,

$$
\lim _{n \rightarrow \infty} \varphi^{n}\left(\frac{s}{a_{n}}\right)=e^{-s^{\alpha}} .
$$

We will now make use of the representation (6) for $\mathrm{E}\left(T_{n}^{k}\right)$. We have to investigate the asymptotic behaviour of $B\left(n, k_{1}, \ldots, k_{r}\right)$. The change of variables $s=t / a_{n}$ together with an application of Potter's theorem [3, Th. 1.5.6], Lebesgue's dominated convergence theorem and Lemma 3.1 leads to

$$
\begin{aligned}
B\left(n, k_{1}, \ldots, k_{r}\right) & =\frac{\left(\begin{array}{l}
n \\
r
\end{array}\right)}{a_{n} \Gamma(2 k)} \int_{0}^{\infty}\left(\frac{t}{a_{n}}\right)^{2 k-1} \varphi^{\left(2 k_{1}\right)}\left(\frac{t}{a_{n}}\right) \cdots \varphi^{\left(2 k_{r}\right)}\left(\frac{t}{a_{n}}\right) \underbrace{\varphi^{n-r}\left(\frac{t}{a_{n}}\right)}_{\rightarrow e^{-t^{\alpha}}} d t \\
& \sim \frac{\alpha^{r}\left(\begin{array}{l}
n \\
r
\end{array}\right)}{a_{n} \Gamma(2 k)} \int_{0}^{\infty}\left(\frac{t}{a_{n}}\right)^{2 k-1}\left(\frac{t}{a_{n}}\right)^{r \alpha-2 k} \ell^{r}\left(\frac{a_{n}}{t}\right)\left(\prod_{j=1}^{r} \Gamma\left(2 k_{j}-\alpha\right)\right) e^{-t^{\alpha}} d t \\
& \sim \frac{\alpha^{r} \prod_{j=1}^{r} \Gamma\left(2 k_{j}-\alpha\right)}{\Gamma(2 k)} \underbrace{\frac{\left(\begin{array}{l}
n \\
r
\end{array}\right) \ell^{r}\left(a_{n}\right)}{a_{n}^{r \alpha}}}_{\rightarrow \Gamma(1-\alpha)^{-r} / r !} \underbrace{\int_{0}^{\infty} t^{r \alpha-1} e^{-t^{\alpha}} d t}_{=(r-1) ! / \alpha} \\
& \sim \frac{\alpha^{r-1} \prod_{j=1}^{r} \Gamma\left(2 k_{j}-\alpha\right)}{r \Gamma(1-\alpha)^{r} \Gamma(2 k)} .
\end{aligned}
$$

Summing over all $r=1, \ldots, k$ in (6), we arrive at

$$
\lim _{n \rightarrow \infty} \mathrm{E}\left(T_{n}^{k}\right)=\frac{k !}{(2 k-1) !} \sum_{r=1}^{k} \frac{\alpha^{r-1}}{r \Gamma(1-\alpha)^{r}} \sum_{\substack{k_{1}, \ldots, k_{r} \geq 1 \\ k_{1}+\cdots+k_{r}=k}} \prod_{j=1}^{r} \frac{\Gamma\left(2 k_{j}-\alpha\right)}{k_{j} !} .
$$

Now observe that

$$
G(r, k):=\sum_{\substack{k_{1}, \ldots, k_{r} \geq 1 \\ k_{1}+\cdots+k_{r}=k}} \prod_{j=1}^{r} \frac{\Gamma\left(2 k_{j}-\alpha\right)}{k_{j} !}
$$

can be determined by generating functions. Concretely, if we look at the $r$-fold product

$$
\left(\Gamma(2-\alpha) x+\frac{\Gamma(4-\alpha)}{2 !} x^{2}+\cdots+\frac{\Gamma(2 m-\alpha)}{m !} x^{m}\right)^{r}
$$


for $m$ sufficiently large, then $G(r, k)$ can be read off as its coefficient of $x^{k}$, since the $k$ th power exactly comprises all contributions of combinations $k_{1}, \ldots, k_{r} \geq 1$ with

$$
k_{1}+\cdots+k_{r}=k
$$

in the above sum. It suffices to choose $m=k-r+1$, since larger powers do not contribute to the coefficient of $x^{k}$ any more. Hence Theorem 3.1 follows from (11).

Remark 3.1. For $k=1$, we obtain $\lim _{n \rightarrow \infty} \mathrm{E}\left(T_{n}\right)=1-\alpha$, which is Theorem 5.3 of [5]. The limit of moments of higher order can now be calculated from (9):

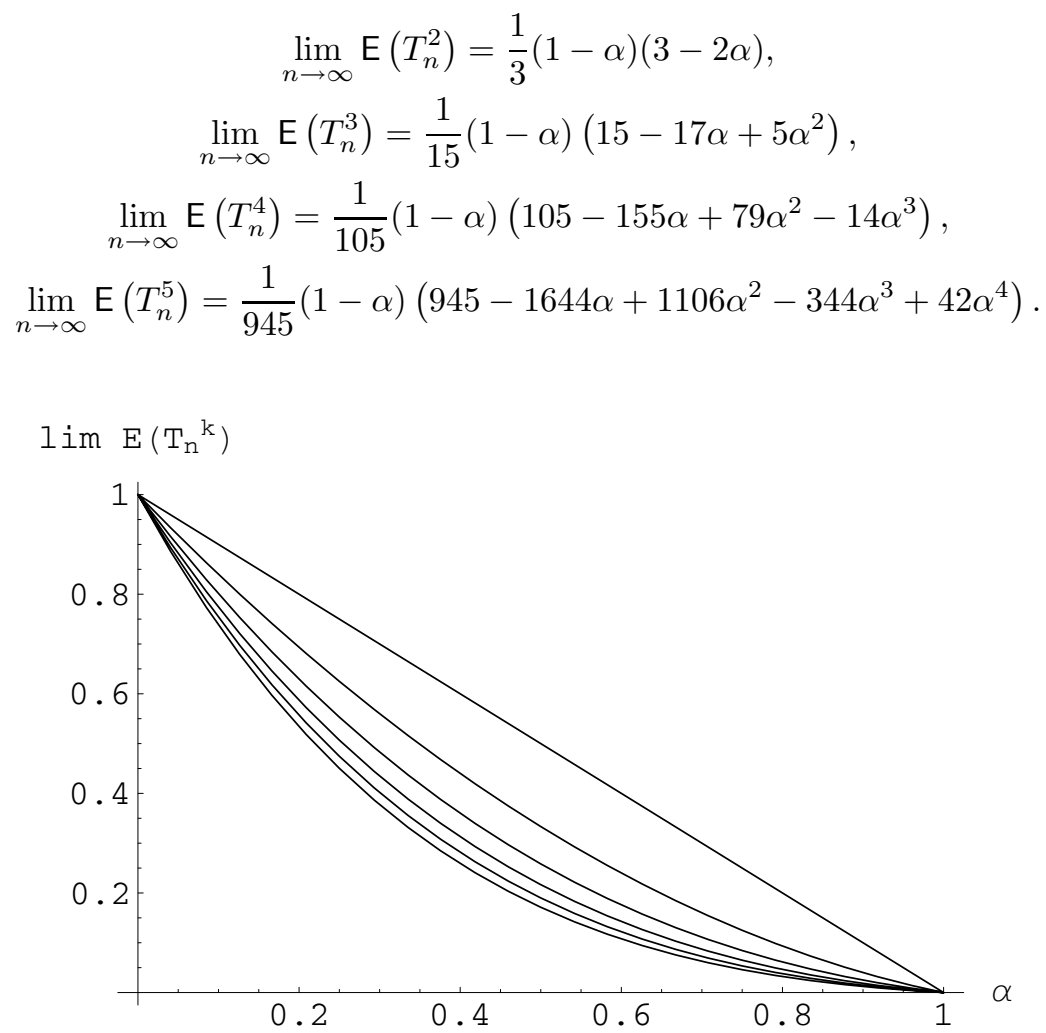

Figure 1. $\lim _{n \rightarrow \infty} \mathrm{E}\left(T_{n}^{k}\right)$ as a function of $\alpha(k=1, \ldots, 5$ from top to bottom)

The following result generalizes Theorem 5.5 of [5], where the case $k=1$ was covered:

Theorem 3.2. If $X_{1}$ belongs to the domain of attraction of a stable law with index $\alpha=1$ and $\mathrm{E}\left(X_{1}\right)=\infty$, then for all $k \geq 1$,

$$
\mathrm{E}\left(T_{n}^{k}\right) \sim \frac{1}{2 k-1} \frac{\ell\left(a_{n}\right)}{\tilde{\ell}\left(a_{n}\right)},
$$

where $\tilde{\ell}(x)=\int^{x}(\ell(t) / t) d t$ and $\left(a_{n}\right)_{n \geq 1}$ is a sequence satisfying $a_{n} \sim n \tilde{\ell}\left(a_{n}\right)$.

Proof. Since $X_{1}$ belongs to the domain of attraction of a stable law with index $\alpha=1$, we have $1-F(x) \sim x^{-1} \ell(x)$ for some slowly varying function $\ell(x)$. Moreover

$$
1-\varphi(s) \sim s \tilde{\ell}\left(\frac{1}{s}\right)
$$


with $\tilde{\ell}(x)=\int^{x}(\ell(t) / t) d t$ (see e.g. [3]). Note that $\tilde{\ell}(x)$ is again a slowly varying function. For any sequence $\left(a_{n}\right)_{n \geq 1}$ with $a_{n} \rightarrow \infty$ we have

$$
\varphi^{n}\left(\frac{s}{a_{n}}\right)=\exp \left\{n \log \varphi\left(s / a_{n}\right)\right\} \sim \exp \left\{-n\left(1-\varphi\left(s / a_{n}\right)\right)\right\} \sim \exp \left\{-n\left(\frac{s}{a_{n}}\right) \tilde{\ell}\left(\frac{a_{n}}{s}\right)\right\} .
$$

If we choose $a_{n}$ such that

$$
n a_{n}^{-1} \tilde{\ell}\left(a_{n}\right) \rightarrow 1 \quad \text { as } n \rightarrow \infty
$$

then

$$
\lim _{n \rightarrow \infty} \varphi^{n}\left(\frac{s}{a_{n}}\right)=e^{-s}
$$

Take $a_{n}$ as in (13) and replace $s$ by $t / a_{n}$ in the representation (6). An application of Potter's theorem, Lebesgue's dominated convergence theorem and Lemma 3.1 yields

$$
\begin{aligned}
B\left(n, k_{1}, \ldots, k_{r}\right) & =\frac{\left(\begin{array}{c}
n \\
r
\end{array}\right)}{a_{n} \Gamma(2 k)} \int_{0}^{\infty}\left(\frac{t}{a_{n}}\right)^{2 k-1} \varphi^{\left(2 k_{1}\right)}\left(\frac{t}{a_{n}}\right) \cdots \varphi^{\left(2 k_{r}\right)}\left(\frac{t}{a_{n}}\right) \underbrace{\varphi^{n-r}\left(\frac{t}{a_{n}}\right)}_{\rightarrow e^{-t}} d t \\
& \sim \frac{\left(\begin{array}{l}
n \\
r
\end{array}\right)}{a_{n} \Gamma(2 k)} \int_{0}^{\infty}\left(\frac{t}{a_{n}}\right)^{2 k-1}\left(\frac{t}{a_{n}}\right)^{r-2 k} \ell^{r}\left(\frac{a_{n}}{t}\right)\left(\prod_{j=1}^{r} \Gamma\left(2 k_{j}-1\right)\right) e^{-t} d t \\
& \sim \frac{\prod_{j=1}^{r} \Gamma\left(2 k_{j}-1\right)}{r ! \Gamma(2 k)} \frac{n^{r} \ell^{r}\left(a_{n}\right)}{a_{n}^{r}} \underbrace{\int_{0}^{\infty} t^{r-1} e^{-t} d t}_{=(r-1) !} \\
& \sim \frac{\prod_{j=1}^{r} \Gamma\left(2 k_{j}-1\right)}{r \Gamma(2 k)}\left(\frac{\ell\left(a_{n}\right)}{\tilde{\ell}\left(a_{n}\right)}\right)^{r} .
\end{aligned}
$$

Note that $\ell\left(a_{n}\right) / \tilde{\ell}\left(a_{n}\right) \rightarrow 0$ for $n \rightarrow \infty$ and thus, opposed to the case $\alpha<1$, only the summand with $r=1$ contributes to the dominating asymptotic term of (6). Therefore we obtain

$$
\mathrm{E}\left(T_{n}^{k}\right) \sim \frac{1}{2 k-1} \frac{\ell\left(a_{n}\right)}{\tilde{\ell}\left(a_{n}\right)} .
$$

Theorem 3.3. Let $X_{1}$ belong to the domain of attraction of a stable law with index $\alpha$, $1 \leq \alpha<2$ and $\mu:=\mathrm{E}\left(X_{1}\right)<\infty$. Then for all $k \geq 1$,

$$
\mathrm{E}\left(T_{n}^{k}\right) \sim \frac{\Gamma(2 k-\alpha) \Gamma(1+\alpha)}{\Gamma(2 k) \mu^{\alpha}} n^{1-\alpha} \ell(n) .
$$

Proof. Since $\mu$ is finite, it follows that

$$
\lim _{n \rightarrow \infty} \varphi^{n}(t / n)=e^{-\mu t} \quad \text { for all } t \geq 0 .
$$

However, in view of (16), we will use the change of variables $s=t / n$ in the representation (6). By virtue of Potter's theorem, Lebesgue's dominated convergence theorem 
and Lemma 3.1 we then obtain

$$
\begin{aligned}
B\left(n, k_{1}, \ldots, k_{r}\right) & =\frac{\left(\begin{array}{c}
n \\
r
\end{array}\right)}{n \Gamma(2 k)} \int_{0}^{\infty}\left(\frac{t}{n}\right)^{2 k-1} \varphi^{\left(2 k_{1}\right)}\left(\frac{t}{n}\right) \cdots \varphi^{\left(2 k_{r}\right)}\left(\frac{t}{n}\right) \underbrace{\varphi^{n-r}\left(\frac{t}{n}\right)}_{\rightarrow e^{-\mu t}} d t \\
& \sim \frac{\alpha^{r}\left(\begin{array}{c}
n \\
r
\end{array}\right)}{n \Gamma(2 k)} \int_{0}^{\infty}\left(\frac{t}{n}\right)^{2 k-1}\left(\frac{t}{n}\right)^{r \alpha-2 k} \ell^{r}\left(\frac{n}{t}\right)\left(\prod_{j=1}^{r} \Gamma\left(2 k_{j}-\alpha\right)\right) e^{-\mu t} d t \\
& \sim \frac{\alpha^{r} \prod_{j=1}^{r} \Gamma\left(2 k_{j}-\alpha\right)}{\Gamma(2 k)} \underbrace{\frac{\left(\begin{array}{c}
n \\
r
\end{array}\right) \ell^{r}(n)}{n^{r \alpha}}}_{\sim n^{r(1-\alpha)} \ell^{r}(n) / r !} \underbrace{\int_{0}^{\infty} t^{r \alpha-1} e^{-\mu t} d t}_{=\Gamma(r \alpha) / \mu^{r \alpha}} \\
& \sim \frac{\alpha^{r} \Gamma(r \alpha) \prod_{j=1}^{r} \Gamma\left(2 k_{j}-\alpha\right)}{r ! \mu^{r \alpha} \Gamma(2 k)} n^{r(1-\alpha)} \ell^{r}(n) .
\end{aligned}
$$

Hence the first-order asymptotic behaviour of (6) is solely determined by the term with $r=1$ and we obtain

$$
\mathrm{E}\left(T_{n}^{k}\right) \sim \frac{\Gamma(2 k-\alpha) \Gamma(1+\alpha)}{\Gamma(2 k) \mu^{\alpha}} n^{1-\alpha} \ell(n)
$$

Remark 3.2. For the special case $k=1$, (15) yields

$$
\mathrm{E}\left(T_{n}\right) \sim \frac{\Gamma(2-\alpha) \Gamma(1+\alpha)}{\mu^{\alpha}} n^{1-\alpha} \ell(n),
$$

which is Theorem 5.1 of $[5$.

We pass to the case $\alpha>2$.

Theorem 3.4. Let $1-F(x) \sim x^{-\alpha} \ell(x)$ for some slowly varying function $\ell(x)$ and $\alpha>2$. Then for all integers $k<\alpha-1$,

$$
\mathrm{E}\left(T_{n}^{k}\right) \sim\left(\frac{\mu_{2}}{\mu^{2}}\right)^{k} n^{-k}
$$

and for $k>\alpha-1$,

$$
\mathrm{E}\left(T_{n}^{k}\right) \sim \frac{\Gamma(2 k-\alpha) \Gamma(1+\alpha)}{\Gamma(2 k) \mu^{\alpha}} n^{1-\alpha} \ell(n)
$$

If $k=\alpha-1$, then

(i) (17) holds if $\ell(x)=o(1)$ (and in particular if $\mathrm{E}\left(X_{1}^{k+1}\right)<\infty$ ),

$$
\mathrm{E}\left(T_{n}^{k}\right) \sim\left(\left(\frac{\mu_{2}}{\mu^{2}}\right)^{k}+C \frac{\Gamma(k-1) \Gamma(k+2)}{\Gamma(2 k) \mu^{1+k}}\right) n^{-k}
$$

holds if $\ell(x) \sim C$ for a constant $C$,

(iii) else (18) holds.

Proof. Let us look at the quantity $B\left(n, k_{1}, \ldots, k_{r}\right)$. By Lemma 3.1 and the BinghamDoney lemma (see e.g. [3, Th. 8.1.6]) the asymptotic behaviour of $\varphi^{(m)}(s)$ at the origin is given by

$$
(-1)^{m} \varphi^{(m)}(s) \sim \begin{cases}\alpha \Gamma(m-\alpha) s^{\alpha-m} \ell(1 / s) & \text { if } m>\alpha, \\ \alpha \tilde{\ell}(1 / s) & \text { if } m=\alpha \text { and } \mathrm{E}\left(X_{1}^{m}\right)=\infty, \\ \mu_{m} & \text { if } m \leq \alpha \text { and } \mathrm{E}\left(X_{1}^{m}\right)<\infty,\end{cases}
$$


where $\tilde{\ell}(x)=\int_{0}^{x}(\ell(u) / u) d u$ is itself a slowly varying function. For simplicity, let us first assume that $\alpha \notin \mathbb{N}$. Then one can conclude in an analogous way as in the proof of Theorem 3.3 that the asymptotic behaviour of $B\left(n, k_{1}, \ldots, k_{r}\right)$ is given by

$$
B\left(n, k_{1}, \ldots, k_{r}\right) \sim C_{1} n^{r-\alpha r_{1}-2\left(k-u_{1}\right)} \ell^{r_{1}}(n),
$$

where $r_{1}$ is the number of integers among $k_{1}, \ldots, k_{r}$ that are greater than $\alpha / 2, u_{1}$ is the sum of these and $C_{1}$ is some constant. It remains to determine the dominating asymptotic term among all possible $B\left(n, k_{1}, \ldots, k_{r}\right)$ : If $r_{1}>0$, then $r_{1}=1, u_{1}=k$ and thus $r=1$ yields the largest exponent, so that the asymptotic order is $n^{1-\alpha} \ell(n)$. Note that $r_{1}>0$ is possible for $2 k>\alpha$ only. For $r_{1}=0$, on the other hand, $r=k$ and thus $k_{1}=\cdots=k_{r}=1$ dominates, leading to asymptotic order $n^{-k}$. Hence the asymptotically dominating power among $B\left(n, k_{1}, \ldots, k_{r}\right)$ is given by $\max (1-\alpha,-k)$. From this we see that for $k<\alpha-1, r=k$ dominates and we obtain from (6),

$$
\mathrm{E}\left(T_{n}^{k}\right) \sim k ! \frac{n^{k} \mu_{2}^{k} \Gamma(2 k)}{k ! \Gamma(2 k) n^{2 k} \mu^{2 k}} \sim\left(\frac{\mu_{2}}{\mu^{2}}\right)^{k} n^{-k} .
$$

Alternatively, if $k>\alpha-1$, the term with $r=1$ dominates and we obtain (18) in just the same way as in Theorem 3.3 .

Finally, the above conclusions also hold for $\alpha \in \mathbb{N}$ except when $k=\alpha-1$. In the latter case the slowly varying function $\ell(x)$ determines which of the two terms $n^{1-\alpha} \ell(n)$ (corresponding to $r=1$ ) and $n^{-k}$ (corresponding to $r=k$ ) dominates the asymptotic behaviour: if $\ell(x)=o(1)$ (which due to $\mathrm{E}\left(X_{1}^{k+1}\right) \sim(k+1) \int_{0}^{n} x^{-1} \ell(x) d x$ is in particular fulfilled for $\left.\mathrm{E}\left(X_{1}^{k+1}\right)<\infty\right)$, the second one dominates. If $\ell(x) \sim$ const, then both terms matter and the assertion of the theorem follows.

Corollary 3.1. If $1-F(x) \sim x^{-2} \ell(x)$, then for $k \geq 2$,

$$
\mathrm{E}\left(T_{n}^{k}\right) \sim \frac{1}{(k-1)(2 k-1) \mu^{2}} \frac{\ell(n)}{n}
$$

and

$$
\mathrm{E}\left(T_{n}\right) \sim \begin{cases}\frac{\mu_{2}}{\mu^{2} n} & \text { if } \mathrm{E}\left(X_{1}^{2}\right)<\infty, \\ \frac{2}{\mu^{2}} \frac{\tilde{\varepsilon}(n)}{n} & \text { if } \mathrm{E}\left(X_{1}^{2}\right)=\infty .\end{cases}
$$

Proof. One can easily verify that Theorem 3.4 remains true for $\alpha=2$ except for $k=1$ in the case $\mathrm{E}\left(X_{1}^{2}\right)=\infty$. In the latter case obviously $r=1$ and one obtains (using $\left.\varphi^{\prime \prime}(s) \sim 2 \tilde{\ell}(1 / s)\right)$

$$
\mathrm{E}\left(T_{n}\right) \sim B(n, 1) \sim \frac{2 n \tilde{\ell}(n)}{n^{2}} \int_{0}^{\infty} t e^{-\mu t} d t \sim \frac{2}{\mu^{2}} \frac{\tilde{\ell}(n)}{n},
$$

which is already contained in [5, Theorem 5.2].

Remark 3.3. One might wonder whether a general limit result for $\mathrm{E}\left(T_{n}^{k}\right)$ for $X_{1}$ in the domain of attraction of a normal law (in the spirit of Theorem 5.2 of $[5$ for $k=1$ ) can be obtained with the integral representation approach used in this paper. This is however not the case: From $\int_{0}^{x} y^{2} d F(y) \sim \ell_{2}(x)$ (where $\ell_{2}(x)$ is a slowly varying function) it follows by partial integration that $\varphi^{(2 k)}(s) / \ell_{2}(1 / s)=o\left(s^{2-2 k}\right)$ for $k>1$ as $s \rightarrow 0$, but the latter is not strong enough to identify the dominating term among the $B\left(n, k_{1}, \ldots, k_{r}\right)$ without any further assumptions on the distribution of $X_{1}$.

As an illustration of the results of this paper, Table 1 gives the first order asymptotic terms of $\mathrm{E}\left(T_{n}\right), \operatorname{Var}\left(T_{n}\right)$ and the dispersion $\operatorname{Var}\left(T_{n}\right) / \mathrm{E}\left(T_{n}\right)$ as a function of $\alpha$. Note that the entries for $\alpha>2$ have been obtained by calculating second-order asymptotic terms. The result for $\alpha>4$ in the table actually holds whenever $\mu_{4}<\infty$, since in this case 
TABLE 1. First order asymptotic terms of $\mathrm{E}\left(T_{n}\right), \operatorname{Var}\left(T_{n}\right)$ and $\operatorname{Var}\left(T_{n}\right) / \mathrm{E}\left(T_{n}\right)$ for $1-F(x) \sim x^{-\alpha} \ell(x)$ as a function of $\alpha$

\begin{tabular}{|c|c|c|c|}
\hline & $\mathrm{E}\left(T_{n}\right)$ & $\operatorname{Var}\left(T_{n}\right)$ & $\operatorname{Var}\left(T_{n}\right) / \mathrm{E}\left(T_{n}\right)$ \\
\hline $0<\alpha<1$ & $1-\alpha$ & $\frac{\alpha(1-\alpha)}{3}$ & $\frac{\alpha}{3}$ \\
\hline$\alpha=1$ & $\left.\frac{\ell\left(a_{n}\right)}{\ell\left(a_{n}\right)} \rightarrow 0\right)$ & $\frac{1}{3} \frac{\ell\left(a_{n}\right)}{\ell\left(a_{n}\right)}(\rightarrow 0)$ & $\frac{1}{3}$ \\
\hline $1<\alpha<2$ & $\frac{\Gamma(2-\alpha) \Gamma(1+\alpha)}{\mu^{2}} n^{1-\alpha} \ell(n)$ & $\frac{\Gamma(4-\alpha) \Gamma(1+\alpha)}{6 \mu^{\alpha}} n^{1-\alpha} \ell(n)$ & $\frac{(3-\alpha)(2-\alpha)}{6}$ \\
\hline$\alpha=2$ & $\frac{2}{\mu^{2}} \frac{\tilde{\ell}(n)}{n}$ & $\frac{\ell(n)}{3 n \mu^{2}}$ & $\frac{1}{6} \frac{\ell(n)}{\bar{\ell}(n)}(\rightarrow 0)$ \\
\hline $2<\alpha<4$ & $\frac{\mu_{2}}{\mu^{2} n}$ & $\frac{\Gamma(4-\alpha) \Gamma(1+\alpha)}{6 \mu^{\alpha}} n^{1-\alpha} \ell(n)$ & $\frac{\Gamma(4-\alpha) \Gamma(1+\alpha)}{6 \mu^{\alpha-2} \mu_{2}} n^{2-\alpha} \ell(n)$ \\
\hline$\alpha \geq 4$ & $\frac{\mu_{2}}{\mu^{2} n}$ & $\frac{\mu_{4} \mu^{2}-\mu_{2}^{2} \mu^{2}+4 \mu_{2}^{3}-4 \mu_{2} \mu_{3}}{\mu^{6}} \frac{1}{n^{3}}$ & $\frac{\mu_{4} \mu^{2} / \mu_{2}-\mu_{2} \mu^{2}+4 \mu_{2}^{2}-4 \mu \mu_{3}}{\mu^{4}} \frac{1}{n^{2}}$ \\
\hline
\end{tabular}

the derivation of second-order terms does not rely on the assumption of regular variation and one obtains

$$
\mathrm{E}\left(T_{n}^{2}\right)=\frac{\mu_{2}^{2}}{\mu^{4}} \frac{1}{n^{2}}+\left(\frac{10 \mu_{2}^{3}-3 \mu_{2}^{2} \mu^{2}-8 \mu \mu_{2} \mu_{3}+\mu^{2} \mu_{4}}{\mu^{6}}\right) \frac{1}{n^{3}}+O\left(\frac{1}{n^{4}}\right)
$$

and

$$
\mathrm{E}^{2}\left(T_{n}\right)=\frac{\mu_{2}^{2}}{\mu^{4}} \frac{1}{n^{2}}+\left(\frac{6 \mu_{2}^{3}-4 \mu \mu_{2} \mu_{3}-2 \mu^{2} \mu_{2}^{2}}{\mu^{6}}\right) \frac{1}{n^{3}}+O\left(\frac{1}{n^{4}}\right) .
$$

From Table 1 we see that the dispersion of $T_{n}$ is a continuous function in $\alpha$ with its maximum in $\alpha=1$ (see Figure 2).

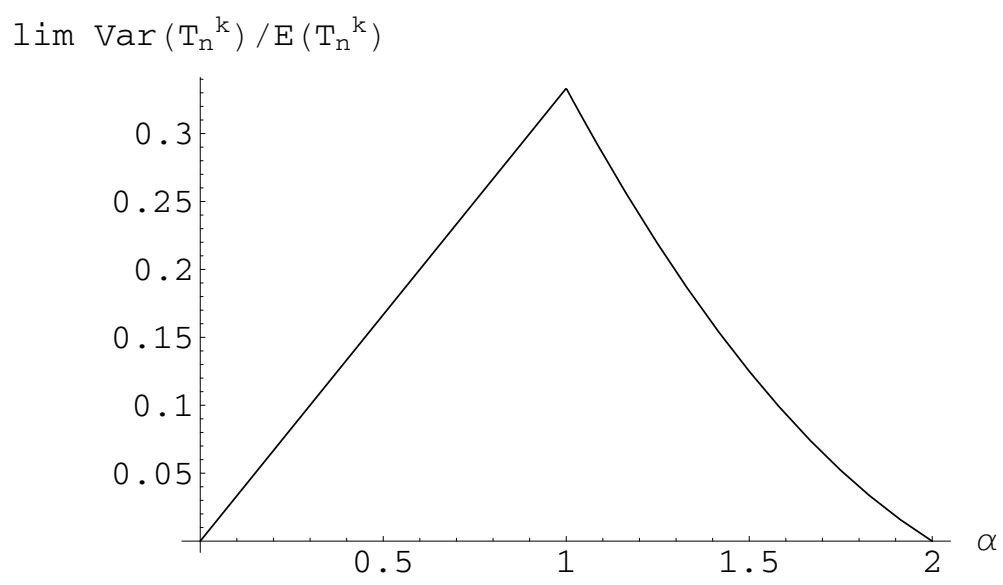

Figure 2. Limit of the dispersion of $T_{n}$ as a function of $\alpha$

\section{Estimation of the extreme value index for Pareto-type tails}

The results of Section 3 also give rise to an alternative and seemingly new method for estimating the extreme value index $1 / \alpha$ for Pareto-type tails $1-F(x) \sim x^{-\alpha} \ell(x)$ with $0<\alpha<2$ from a given data set of independent observations (see e.g. 2] for other 
estimators of the extreme value index). In fact, plotting $n T_{n}$ against $n$ will tend to a line with slope $1-\alpha$, if $0<\alpha<1$ and plotting $\log \left(n T_{n}\right)$ against $\log n$ will tend to a line with slope $2-\alpha$, if $1<\alpha<2$. The asymptotic behaviour of higher order moments of $n T_{n}$ available from Section 3 can then be used to increase the efficiency of the estimation procedure.

At the same time, this provides a technique to test the finiteness of the mean of a distribution in the domain of attraction of a stable law.

Acknowledgement. The authors would like to thank Sophie Ladoucette for a careful reading of the manuscript.

\section{BIBLIOGRAPHY}

1. H. Albrecher, S. Ladoucette, and J. Teugels, Asymptotics of the Sample Coefficient of Variation and the Same Dispersion, K. U. Leuven UCS Report 2006-04, 2006.

2. J. Beirlant, Y. Goegebeur, J. Segers, and J. Teugels, Statistics of Extremes: Theory and Applications, Wiley, Chichester, 2004. MR2108013 (2005j:62002)

3. N. Bingham, C. Goldie, and J. Teugels, Regular variation, Encyclopedia of Mathematics and its Applications, vol. 27, Cambridge University Press, Cambridge, 1987. MR898871 (88i:26004)

4. H. Cohn and P. Hall, On the limit behaviour of weighted sums of random variables, Z. Wahrsch. Verw. Gebiete 59 (1982), no. 3, 319-331. MR721629 (85g:60029)

5. A. Fuchs, A. Joffe, and J. Teugels, Expectation of the ratio of the sum of squares to the square of the sum: exact and asymptotic results, Theory Probab. Appl. 46 (2001), no. 2, 243-255. MR:1968687 (2004b:62045)

6. D. L. McLeish and G. L. O'Brien, The expected ratio of the sum of squares to the square of the sum, Ann. Probab. 10 (1982), no. 4, 1019-1028. MR672301 (84a:60039)

Department of Mathematics, Graz University of Technology, Steyrergasse 30, A-8010 Graz, Austria

E-mail address: albrecher@TUGraz.at

Department of Mathematics, Katholieke Universiteit Leuven, W. De Croylaan 54, B-3001 Heverlee, Belgium, and Eurandom, P.O. Box 513 - 5600 MB Eindhoven, The Netherlands

E-mail address: jef.teugels@wis.kuleuven.ac.be

Received 1/FEB/2005

Originally published in English 